\title{
Assessment of the near infrared identification of carbon stars
}

\section{The local group galaxies IC 1613 and NGC 3109 ${ }^{\star \star \star \star}$}

\author{
P. Battinelli ${ }^{1}$ and S. Demers ${ }^{2}$ \\ 1 INAF, Osservatorio Astronomico di Roma Viale del Parco Mellini 84, 00136 Roma, Italy \\ e-mail: battinel@inaf.it \\ 2 Département de Physique, Université de Montréal, C.P. 6128, Succursale Centre-Ville, Montréal, Qc, H3C 3J7, Canada \\ e-mail: demers@astro.umontreal.ca
}

Received 16 July 2008 / Accepted 3 October 2008

\section{ABSTRACT}

\begin{abstract}
Context. Thousands of $\mathrm{C}$ stars have been identified in Local Group galaxies using narrow band photometry. To survey $\mathrm{C}$ stars at larger distances and alternative to the narrow-band approach is needed.

Aims. We obtain, from ESO Archive data, NIR magnitudes and colours of previously known C stars in two dwarf irregular Local Group galaxies, namely IC 1613 and NGC 3109.

Methods. We compare the NIR magnitudes and colours of the $\mathrm{C}$ star populations, previously identified from narrow band techniques to estimate the star formation history of their intermediate-age populations.

Results. We demonstrate that the photometric properties of $\mathrm{C}$ stars show a wider range in the NIR than in the optical. As confirmed by models we see, among the four galaxies investigated with a 0.7 dex metallicity range, little if any metallicity effect in the $\mathrm{C}$ star colour distribution.
\end{abstract}

Key words. stars: AGB and post-AGB - stars: carbon - galaxies: dwarf

\section{Introduction}

In a previous paper (Battinelli et al. 2007, hereafter Paper I) we compiled near-infrared mean magnitudes and colours of carbon stars, mostly identified from narrow-band photometry, in various galaxies. We concluded that $\left(J-K_{\mathrm{s}}\right)_{0}=1.4$ can be regarded as a conservative limit for the selection of $\mathrm{C}$ stars among the AGB population because essentially no M-type stars have such a red colour. This colour limit should not, however, be used to count $\mathrm{C}$ and M-type AGB stars because too many $\mathrm{C}$ stars do have colours bluer than the above limit and near-infrared colours are not useful to establish the $\mathrm{C} / \mathrm{M}$ ratio in galaxies. Nevertheless, the NIR approach to AGB studies does appear to be a promising tool to reach distant galaxies since most of the energy output of these late-type giants is in the red.

In order to further compare the NIR properties of C stars and assess their usefulness as near-infrared standard candles, we continue our survey of nearby galaxies. To do so, we searched the ESO archives for SOFI or ISAAC observations of Local Group galaxies. We present here NIR magnitudes and colours of previously known C stars in IC 1613 and NGC 3109.

\subsection{The target galaxies}

Luminosity wise, with $M_{v}=-14.9$, IC 1613 ranks 6th among the 15 dwarf irregular (dIrr) galaxies of the Local Group, being between NGC 3109 and Sextans A. Its distance has been accurately determined by Dolphin et al. (2001), from the apparent

\footnotetext{
* This publication makes use of data products from the ESO Archive.

$\star \star$ Full Tables 2 and 3 are only available in electronic form at http://www . aanda.org
}

magnitudes of the red giant branch tip (TRGB) and of the red clump stars, who obtained $\mu_{\mathrm{o}}=24.31 \pm 0.06(730 \pm 20 \mathrm{kpc})$. Pietrzyński et al. (2006a) determined the IC 1613 distance to be $\mu_{\mathrm{o}}=24.291 \pm 0.014(720 \pm 5 \mathrm{kpc})$, from near infrared photometry of its Cepheids. Based on these two estimates, we adopt $722 \pm 5 \mathrm{kpc}$ for the distance of IC 1613. The foreground reddening is taken to be $E(B-V)=0.03$, corresponding to $E\left(J-K_{\mathrm{s}}\right)=0.016$. IC 1613 is essentially a typical irregular galaxy. Albert et al. (2000), using the narrow-band index (CN - TiO), identified some $200 \mathrm{C}$ stars extending to $15^{\prime}$ from the center of IC 1613. This result not surprising since the NIR observations of IC 1613 obtained by Borissova et al. (2000) revealed that the galaxy has a rich intermediate-age AGB population. According to Skillman et al. (2003), IC 1613 has a relatively constant star formation rate over the age of the Universe.

Recently, Zucker \& Wyder (2004), using DEIMOS at the Keck telescope, determined the metal abundance of numerous RGB stars in IC 1613. The mean abundance, based on the equivalent width of the CaII triplet, is quoted as $[\mathrm{Fe} / \mathrm{H}]=-1.3$. This value is in good agreement with the Skillman et al. (2003) SFH models which suggest an abundance of $[\mathrm{Fe} / \mathrm{H}] \approx-1.0$ for the intermediate-age stars.

NGC 3109, an outlying member of the Local Group, is a southern hemisphere dwarf irregular galaxy, more luminous and more massive than IC 1613 . Because of its proximity to us it turns out to be one of the brightest galaxies seen in the southern sky. Its carbon star population has been surveyed by Demers et al. (2003) who identified over $400 \mathrm{C}$ stars using the narrow band technique. Recently the near-infrared $J$ and $K_{\mathrm{s}}$ magnitudes of 77 Cepheids have been used by Soszyński et al. (2006) to accurately determine the distance of NGC 3109 . We adopt their estimates, $(m-M)_{0}=25.57 \pm 0.024$ along with $E(B-V)=0.09$, 
Table 1. Fields observed in each galaxy.

\begin{tabular}{lcc}
\hline \hline Galaxy & \multicolumn{2}{c}{ RA J2000 Dec } \\
\hline IC 1613 F1 & $01: 04: 57.0$ & $+02: 08: 36$ \\
IC 1613 F2 & $01: 04: 40.5$ & $+02: 08: 37$ \\
IC 1613 F3 & $01: 04: 52.3$ & $+02: 03: 50$ \\
NGC 3109 F1 & $10: 03: 18.3$ & $-26: 09: 18$ \\
NGC 3109 F2 & $10: 03: 08.0$ & $-26: 10: 10$ \\
NGC 3109 F3 & $10: 02: 58.4$ & $-26: 09: 53$ \\
\hline
\end{tabular}

or $E\left(J-K_{\mathrm{s}}\right)=0.048$ and $A_{K}=0.03$. Alonso et al. (1999) published NIR observations of NGC 3109 . However, their data are not deep enough for a full assessment of the intermediate-age population. The metallicity of the old population of NGC 3109 has been estimated by Mendez et al. (2002) from the $(V-I)_{0, \mathrm{TRGB}}$ to be $[\mathrm{Fe} / \mathrm{H}]=-1.69 \pm 0.06$.

\section{Observations and data reduction}

\subsection{IC 1613}

The $J$ and $K_{\mathrm{s}}$ observations of IC 1613 were obtained from the ESO Archive. They consist of NTT data, from La Silla, obtained with the SOFI near infrared camera in September 2004 as part of the Araucaria Cepheid project (Pietrzyński et al. 2006a). The field of SOFI is $4.9^{\prime} \times 4.9^{\prime}$ and the scale is 0.288 arcsec per pixel. The coordinates of the three fields, read from the image header, are given in Table 1. Details of these observations are described by Pietrzyński et al. (2006a). In the $K_{\mathrm{s}}$ filter, they obtained six consecutive $10 \mathrm{~s}$ integrations at a given position and then moved the telescope by about $20^{\prime \prime}$ to a different random position. Integrations obtained at 65 different dithering positions resulted in a total exposure time of $65 \mathrm{~min}$ in the $K_{\mathrm{s}}$ filter for a given field. For the $J$ filter for which the sky shows less variation, two consecutive $20 \mathrm{~s}$ exposures were obtained for 25 dithering positions for a total of 17 min on a given field.

The raw images obtained from the Archives were reduced using the XDIMSUM task in IRAF. The resulting combined images were analysed with DAOPHOT and ALLSTAR. After obtaining the astrometric solution for one image in each field we match the stars found with the 2MASS catalogue. The zero point adjustments between our instrumental magnitudes and the 2MASS magnitudes are based on about six stars per field, excluding the fainter 2MASS stars with large uncertainties. The standard deviations of the differences between the 2MASS magnitudes and our instrumental magnitudes are \pm 0.08 for $K_{\mathrm{s}}$ and \pm 0.07 for $J$. The $J$ zero points for the three fields as well as those in $K_{\mathrm{s}}$ of F1 and F2 agree within the errors, while the $K_{\mathrm{s}}$ zero point of $\mathrm{F} 3$ differs by $0.6 \mathrm{mag}$.

Further assessment of the reliability of our photometry can be done by comparing the magnitudes of the stars in the overlapping region between field $\mathrm{F} 1$ and $\mathrm{F} 2$. We calculate, for 45 stars, $\Delta K_{\mathrm{s}}=-0.06 \pm 0.10$; a somewhat larger difference is found for $J$ but with the same dispersion.

\subsection{NGC 3109}

The NGC 3109 images are also extracted from the ESO Archives. They were obtained with ISAAC at the VLT by Soszyński et al. (2006) as part of the Araucaria project. They observed three $2.5^{\prime} \times 2.5^{\prime}$ fields in $J$ and $K_{\mathrm{s}}$ bands; the scale is $0.147^{\prime \prime}$ per pixel. The reader is referred to the above publication for further observation details. We reduce the raw images

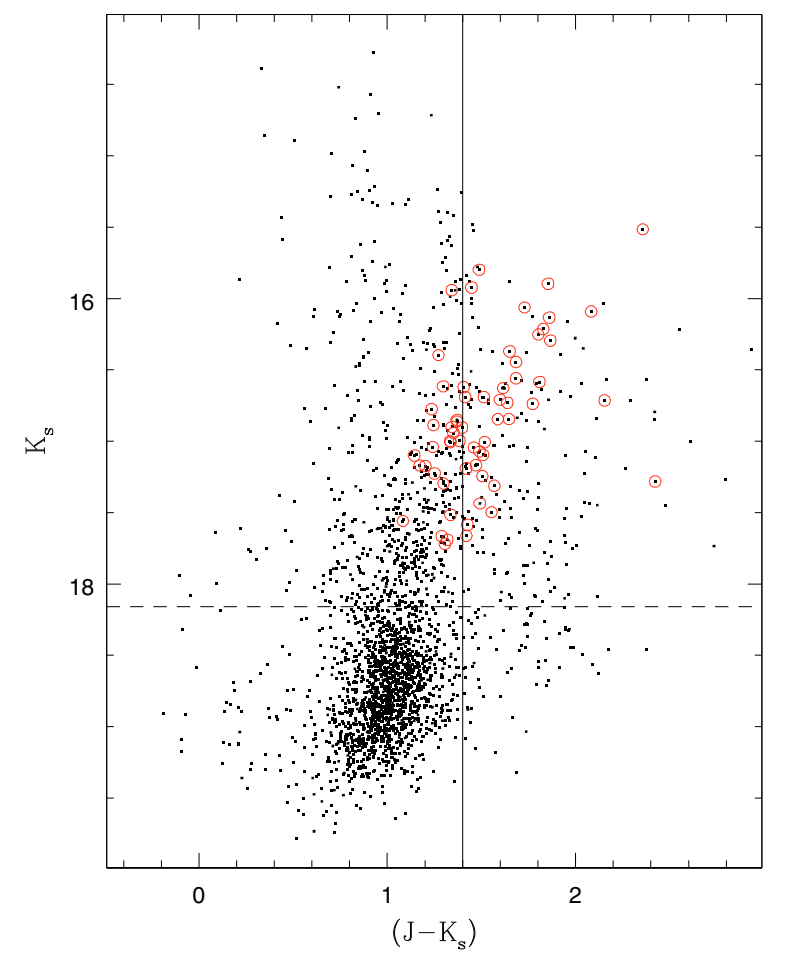

Fig. 1. The colour-magnitude diagram of the combined three IC 1613 fields. The circles correspond to the known $\mathrm{C}$ stars identified from narrow-band photometry. The dashed line is at the TRGB level and the vertical lines marks the $J-K_{\mathrm{s}}=1.4$ limit.

using JITTER from the ECLIPSE package developed by ESO. The combined images were then analysed with DAOPHOT and ALLSTAR.

The small size of the images makes the astrometric and magnitude calibrations more difficult than for the SOFI data. For the astrometry we use as a reference the $\mathrm{C}$ star coordinates published by Demers et al. (2003) and seen in the field. Because of the lack of suitable 2MASS stars, the magnitude calibration is done using the published $J$ and $K_{\mathrm{S}}$ magnitudes of the Cepheids (Soszyński et al. 2006), taken on the same night as the images we analyse. The Cepheid coordinates are listed by Pietrzyński et al. (2006b). We first adjust the magnitude zero points of field $\mathrm{F} 1$ and $\mathrm{F} 3$ to those of F2 by comparing the magnitudes of stars in the overlapping regions. We then use the published magnitudes of 36 Cepheids with $K_{\mathrm{s}}<20.0$ seen within the three fields to calibrate the instrumental magnitudes. From this comparison we obtain $\sigma_{K}= \pm 0.18$ and $\sigma_{J}= \pm 0.09 \mathrm{mag}$. The $\mathrm{J} 2000$ equatorial coordinates of the NGC 3109 fields are given in Table 1 .

\section{Results}

\subsection{IC 1613}

The colour-magnitude diagram of the combined three SOFI fields of IC 1613 is displayed in Fig. 1. Sixty one C stars identified by Albert et al. (2000) are matched to the stars above the TRGB in our NIR photometry. Their NIR magnitude and colour are given in Table 2. The identification numbers are those assigned by Albert et al. (2000). Among the 61 stars matched, only $37(61 \%)$ of them have $\left(J-K_{\mathrm{s}}\right)>1.4$. If we assume that all AGB stars above the TRGB and with $\left(J-K_{\mathrm{S}}\right)>1.4$ are $\mathrm{C}$ stars, then there are 190 such objects in our IC 1613 fields. 
Table 2. NIR magnitudes and colours of C stars in IC $1613^{a}$.

\begin{tabular}{ccccccc}
\hline \hline id & RA & Dec & $K_{\mathrm{s}}$ & $\sigma_{K}$ & $J-K_{\mathrm{s}}$ & $\sigma_{J-K}$ \\
\hline 39 & $1: 04: 30.50$ & $2: 06: 47.7$ & 17.516 & 0.015 & 1.336 & 0.021 \\
49 & $1: 04: 33.90$ & $2: 08: 31.3$ & 15.941 & 0.009 & 1.342 & 0.017 \\
50 & $1: 04: 34.00$ & $2: 06: 00.6$ & 16.627 & 0.012 & 1.615 & 0.021 \\
51 & $1: 04: 34.50$ & $2: 10: 09.5$ & 16.999 & 0.012 & 1.333 & 0.018 \\
54 & $1: 04: 35.30$ & $2: 06: 06.1$ & 16.942 & 0.012 & 1.352 & 0.020 \\
55 & $1: 04: 35.40$ & $2: 06: 54.4$ & 16.904 & 0.011 & 1.397 & 0.016 \\
56 & $1: 04: 35.60$ & $2: 08: 31.3$ & 16.371 & 0.009 & 1.650 & 0.017 \\
57 & $1: 04: 36.60$ & $2: 06: 39.7$ & 16.134 & 0.009 & 1.861 & 0.014 \\
60 & $1: 04: 37.10$ & $2: 07: 47.4$ & 17.244 & 0.013 & 1.505 & 0.019 \\
62 & $1: 04: 37.40$ & $2: 07: 39.9$ & 17.168 & 0.011 & 1.471 & 0.018 \\
63 & $1: 04: 37.40$ & $2: 09: 40.1$ & 17.006 & 0.012 & 1.336 & 0.021 \\
\hline
\end{tabular}

${ }^{a}$ Table 2 is presented in its entirety in the electronic edition of the journal. A portion is shown here for guidance regarding its form and content.

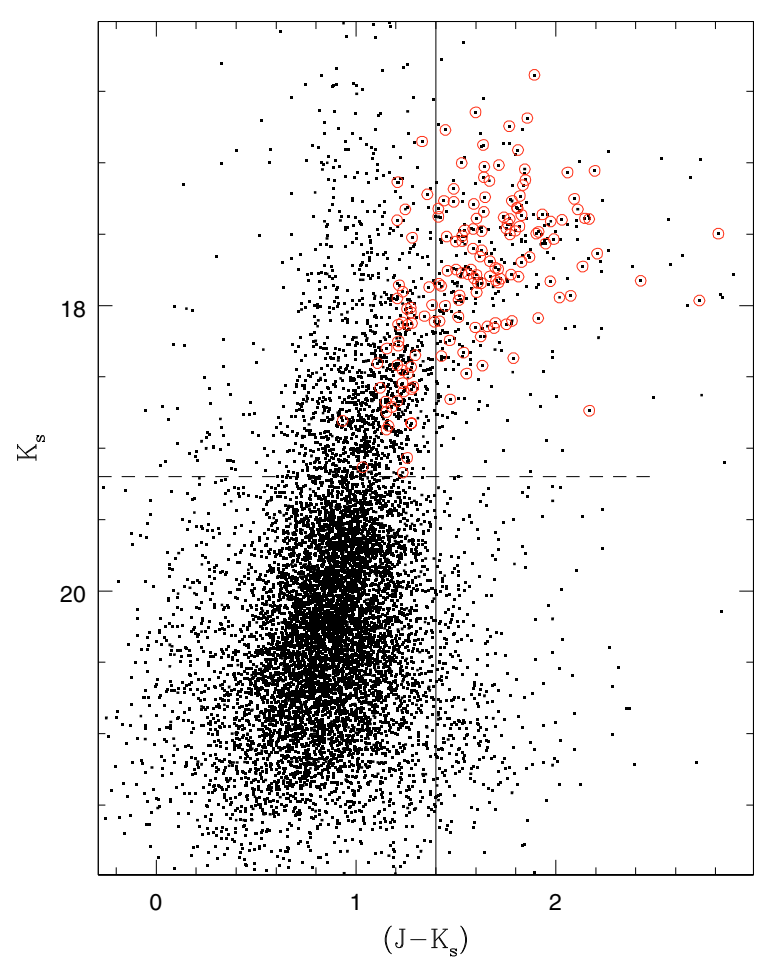

Fig. 2. CMD of the three fields of NGC 3109. The dashed line traces the level of the TRGB and the vertical lines is at $\left(J-K_{\mathrm{s}}\right)=1.4$. The matched $\mathrm{C}$ stars are circled.

\subsection{NGC 3109}

The colour magnitude diagram of the combined three ISAAC fields of NGC 3109 is presented in Fig. 2. 157 C stars were crossidentified with our optical photometry (Demers et al. 2003); a few stars are found in more than one ISAAC fields. They are identified by circles on the CMD of Fig. 2. We list, in Table 3, the NIR magnitude and colour of the $\mathrm{C}$ stars. The identification numbers are from Demers et al. (2003). Among the 157 stars matched, 111 of them $(71 \%)$ have $\left(J-K_{\mathrm{s}}\right)>1.4$ If, similarly to IC 1613 , we call all the AGB redder than $1.4 \mathrm{C}$ stars, then we count 310 such stars in the NGC 3109 fields. The interpretation of these numbers is difficult. For example, a comparison of Figs. 1 and 2 reveals an abnormal number of faint red stars in the IC 1613 CMD, at the TRGB level, not seen in NGC 3109. The nature of the stars with $\left(J-K_{\mathrm{S}}\right)>1.4$ cannot be confirmed
Table 3. NIR magnitudes and colours of C stars in NGC $3109^{a}$.

\begin{tabular}{lcccccc}
\hline \hline id & RA & Dec & $K_{\mathrm{s}}$ & $\sigma_{K}$ & $J-K_{\mathrm{s}}$ & $\sigma_{J-K}$ \\
\hline 106 & $10: 02: 53.17$ & $-26: 09: 08.2$ & 18.023 & 0.031 & 1.255 & 0.048 \\
108 & $10: 02: 53.92$ & $-26: 10: 20.1$ & 17.550 & 0.037 & 1.531 & 0.056 \\
109 & $10: 02: 54.11$ & $-26: 10: 33.2$ & 17.514 & 0.043 & 1.532 & 0.056 \\
111 & $10: 02: 54.29$ & $-26: 09: 57.8$ & 18.843 & 0.040 & 1.163 & 0.047 \\
112 & $10: 02: 54.59$ & $-26: 10: 02.5$ & 18.081 & 0.025 & 1.513 & 0.029 \\
113 & $10: 02: 54.74$ & $-26: 08: 52.1$ & 18.477 & 0.035 & 1.260 & 0.043 \\
116 & $10: 02: 54.90$ & $-26: 10: 34.8$ & 18.586 & 0.038 & 1.278 & 0.048 \\
117 & $10: 02: 55.24$ & $-26: 09: 13.0$ & 18.302 & 0.023 & 1.154 & 0.026 \\
118 & $10: 02: 55.40$ & $-26: 09: 19.2$ & 18.868 & 0.033 & 1.153 & 0.038 \\
119 & $10: 02: 55.42$ & $-26: 10: 59.8$ & 17.997 & 0.038 & 1.382 & 0.054 \\
122 & $10: 02: 55.95$ & $-26: 08: 37.6$ & 18.403 & 0.045 & 1.108 & 0.060 \\
123 & $10: 02: 56.59$ & $-26: 08: 54.7$ & 17.477 & 0.021 & 1.797 & 0.028 \\
\hline
\end{tabular}

${ }^{a}$ Table 3 is presented in its entirety in the electronic edition of the journal. A portion is shown here for guidance regarding its form and content.

by our optical photometry because we do not have equatorial coordinates for either of the full databases.

\section{Discussion}

\section{1. $C$ stars in the $C M D$}

Since the regions observed in NGC 3109 and IC 1613 contain numerous Cepheids we must conclude that we are dealing with populations of mixed ages. In Fig. 3 we draw the Cioni et al. (2006) published isochrones of AGB models on the colourmagnitude diagram of the known $\mathrm{C}$ stars in IC 1613. We prefer these isochrones to those of Marigo et al. (2008) because the latter do not reach $\left(J-K_{\mathrm{s}}\right)$ colours as red as the $\mathrm{C}$ stars observed. We select a metallicity of $Z=0.001$, the most appropriate for the adopted abundance. The youngest $\mathrm{C}$ stars have an age of $\sim 400$ Myr while the bulk of the $\mathrm{C}$ star population is between $700 \mathrm{Myr}$ and 2.5 Gyr. Some faint C stars can be explained by a lower metallicity as shown by the dashed line (corresponding to $2.5 \mathrm{Gyr}$ and $z=0.0004$ ). A similar plot for NGC 3109, with its adopted lower metallicity, is presented in Fig. 4. Here we see that all C stars were formed between 250 Myr and 2.5 Gyr ago. Again we note that SFH has been variable during that interval. More than half the $\mathrm{C}$ stars are between 500 and 800 Myr old.

The $\mathrm{C}$ star population in the galaxies discussed in Paper I can likewise be compared, selecting the proper abundances. We display in Fig. 5 the CMDs of C stars in IC 10, NGC 6822 and WLM. The NGC 6822 data are taken from Kang et al. (2006). The SFH of the intermediate age population is similar in the three galaxies with the oldest star being some 2.5 Gyr old and the youngest close to half a Gyr.

\subsection{Colour distribution of $C$ stars}

We remarked in Paper I that $\mathrm{C}$ stars are found over a wide range of NIR colours. We compare, in Fig. 6, the $\left(J-K_{\mathrm{s}}\right)_{0}$ colour distributions of $\mathrm{C}$ and $\mathrm{M}$ stars, previously identified from our RICNTiO photometry in four dwarf galaxies: IC 1613 and NGC 3109 which NIR are presented here, and WLM from Paper I and NGC 6822 with NIR from Kang et al. (2006). We omit here IC 10 because of its extreme and ill-defined colour excess. Stars with positive $(\mathrm{CN}-\mathrm{TiO})$ are $\mathrm{C}$ stars while those with a negative index are $\mathrm{M}$ stars which could be giants in the galaxy or dwarf $M$ stars seen along the line of sight. The 


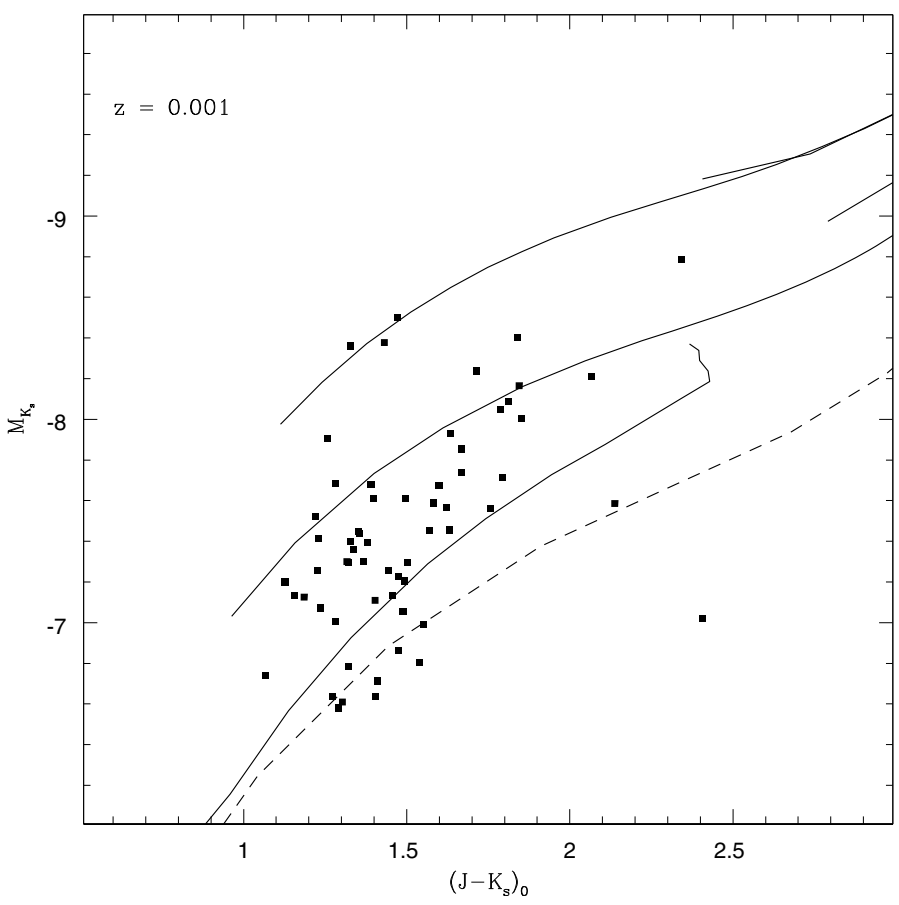

Fig. 3. CMD of known C stars in IC 1613. The isochrones of Cioni et al. (2006) indicate that $\mathrm{C}$ stars were formed between $400 \mathrm{Myr}$ and $2.5 \mathrm{Gyr}$ ago. The bulk of the $\mathrm{C}$ stars were formed more than $700 \mathrm{Myr}$ ago. The dashed isochrone corresponds to $z=0.0004$.

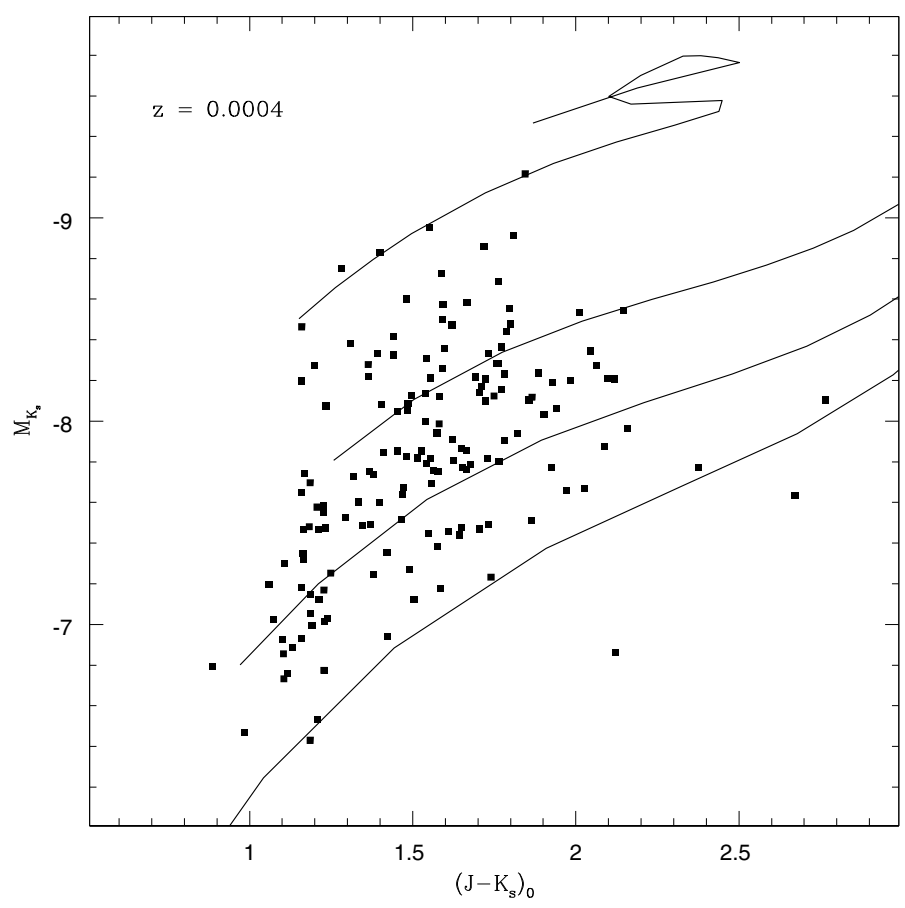

Fig. 4. CMD of known $\mathrm{C}$ stars in NGC 3109. The isochrones correspond, from top to bottom, to ages of $0.25,0.5,0.8$ and $2.5 \mathrm{Gyr}$.

narrow band technique can, however, discriminate only stars with $(R-I)>0.9$, a colour limit corresponding to N-type C stars. We adopted, in Paper I, $\left(J-K_{\mathrm{s}}\right)_{0}=1.40$ as the working limit to separate $\mathrm{C}$ and $\mathrm{M}$ stars. We see, from the vertical lines drawn in Fig. 6, that this limit corresponds essentially to the red border of the $\mathrm{M}$ stars but certainly not to the blue border of $\mathrm{C}$ stars.

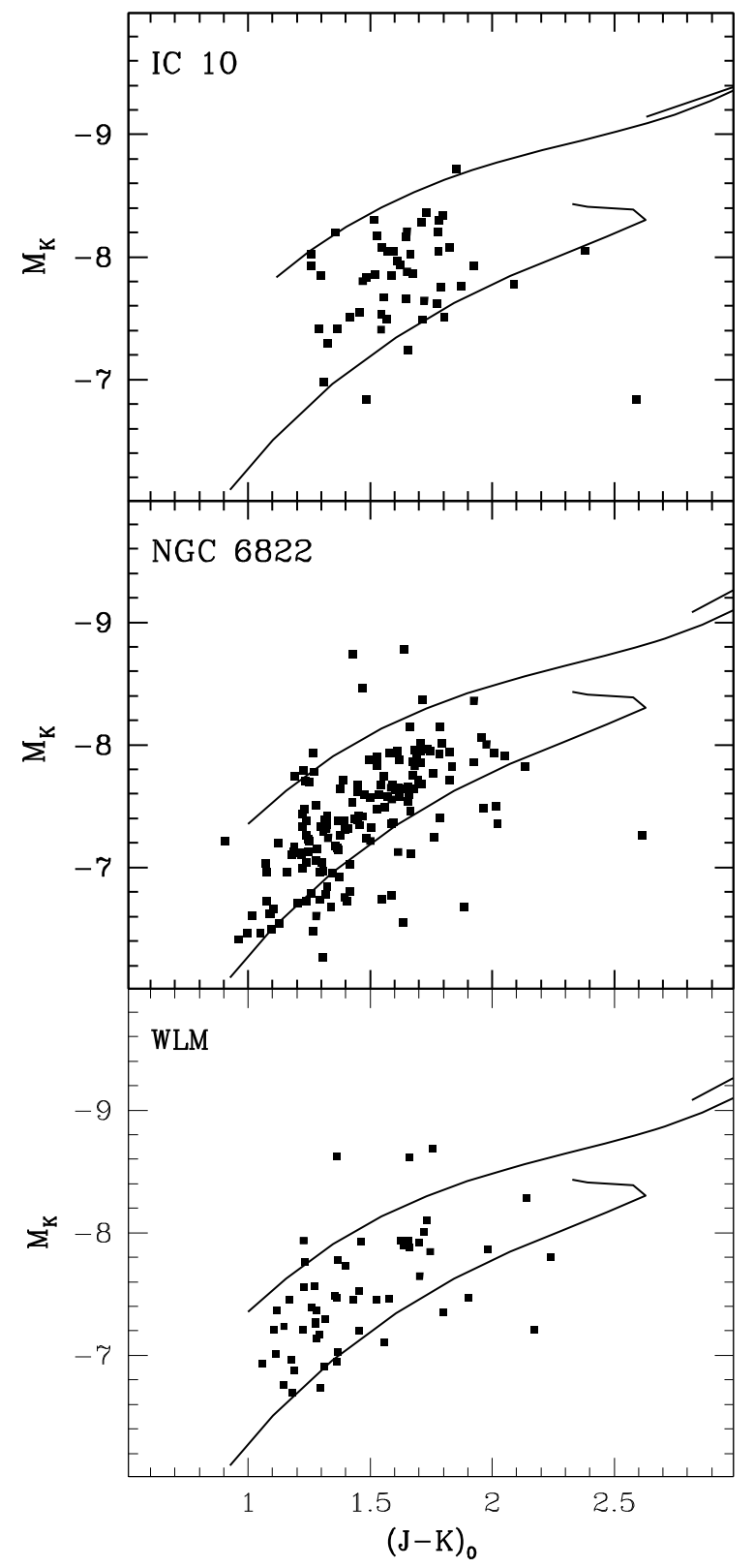

Fig. 5. CMD of known C stars in IC 10, NGC 6822 and WLM. The lower isochrones correspond to $2.5 \mathrm{Gyr}$ while the top ones represent, respectively, $450 \mathrm{Myr}$ (IC 10), $560 \mathrm{Myr}$ (NGC 6822) and $560 \mathrm{Myr}$ (WLM).

Various authors (Davidge 2003; Cioni \& Habing 2003; Cioni \& Habing 2005; Valcheva et al. 2007) have shifted the blue limit of $\mathrm{C}$ stars to take into account the metallicity of the galaxy under consideration. The metallicity of the intermediate-age populations of Local Group galaxies is in general rather poorly known. High resolution spectroscopy has recently target young giants in a few LG dwarf irregulars while the equivalent width of the CaII triplet has been used to infer the abundance of old red giants. The intermediate-age populations of the four galaxies displayed in Fig. 6 show a rather narrow range of metallicities. We adopted earlier for IC 1613 and NGC 3109 respectively $[\mathrm{Fe} / \mathrm{H}]=-1.0$ and -1.69 , while Battinelli \& Demers (2005) adopted -1.4 for WLM and -1.25 for NGC 6822 . The $C$ star distributions in the $(\mathrm{CN}-\mathrm{TiO})-\left(J-K_{\mathrm{s}}\right)$ plane are surprisingly similar, with possibly an exception in the case of NGC 6822 where a "blue C star branch" is seen going toward $(\mathrm{CN}-\mathrm{TiO})=0.0$. The numbers 


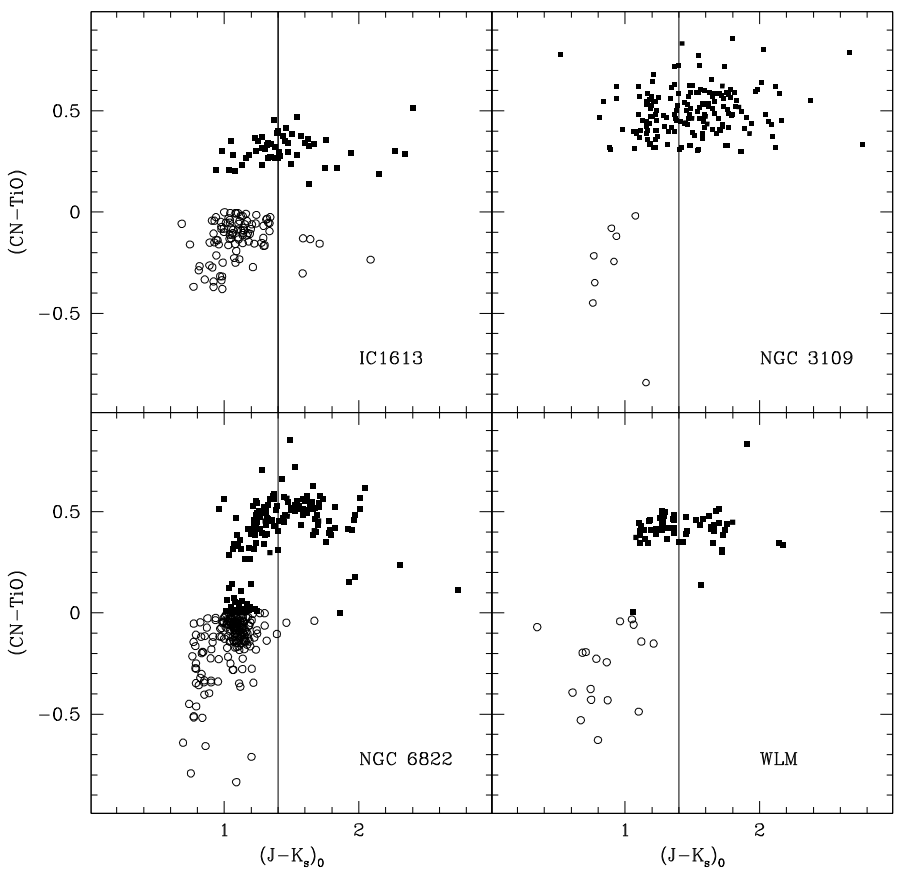

Fig. 6. C (filled squares) and $M$ stars (open circles) as defined from RICNTiO photometry have NIR colours that overlap. The vertical lines trace the $\left(J-K_{\mathrm{s}}\right)_{0}=1.4$ limit.

of C stars plotted depend on the size and stellar density of the regions surveyed. Thus, we see little metallicity effect here.

\subsection{C stars on the colour-colour diagram}

Whitelock et al. (2006) have investigated the NIR colours of Galactic and Magellanic C stars. They conclude that individual members of the two populations cannot be distinguished from their position in the $(J-H)$ vs. $\left(H-K_{\mathrm{s}}\right)$ plane. We do not have $H$ magnitudes for the $C$ stars observed in NGC 3109 and IC 1613 but such data are available for IC 10, WLM and NGC 6822 discussed in Paper I. We are then in a position to compare their locations on the colour-colour diagram. The NIR colours of known $\mathrm{C}$ stars identified from the $(\mathrm{CN}-\mathrm{TiO})$ index in these three galaxies are plotted in Fig. 7. The dashed line is the Whitelock et al. (2006) regression. We draw a representative isochrone from Marigo et al. (2008) for $1 \mathrm{Gyr}, z=0.001$. Isochrones of different metallicities nearly coincide, proving that there is no appreciable shift with abundance. We observe that the data points of WLM and IC 10 essentially overlap, while those of NGC 6822 are offset. Since NGC 6822 contains numerous $\mathrm{C}$ stars it would be useful to obtain an independent dataset to confirm this shift.

Jackson et al. (2007a) in their Spitzer observations of WLM state that only $18 \%$ of the AGB C stars were detected by the $(\mathrm{CN}-\mathrm{TiO})$ approach. This number is somewhat misleading; as explained later by Jackson et al. (2007b), the narrow band technique can only detect the subsample $C$ stars with $(R-I)>0.90$ that corresponds to the N-types. In Fig. 8 we cross identify our NIR data of the AGB stars of IC 1613 with the Spitzer data to produce a colour-colour diagram. The $\mathrm{C}$ stars identified from their (CN - TiO) index by Albert et al. (2000) are also cross identified with the NIR observations. We see that there are, as expected, few $\mathrm{C}$ stars bluer than $\left(J-K_{\mathrm{S}}\right) \approx 1.4$. There are also few $C$ stars among the AGBs that have a positive [3.6] - [4.5] Spitzer index, presumably because these stars were too faint in the optical to be included in the Albert et al. list.

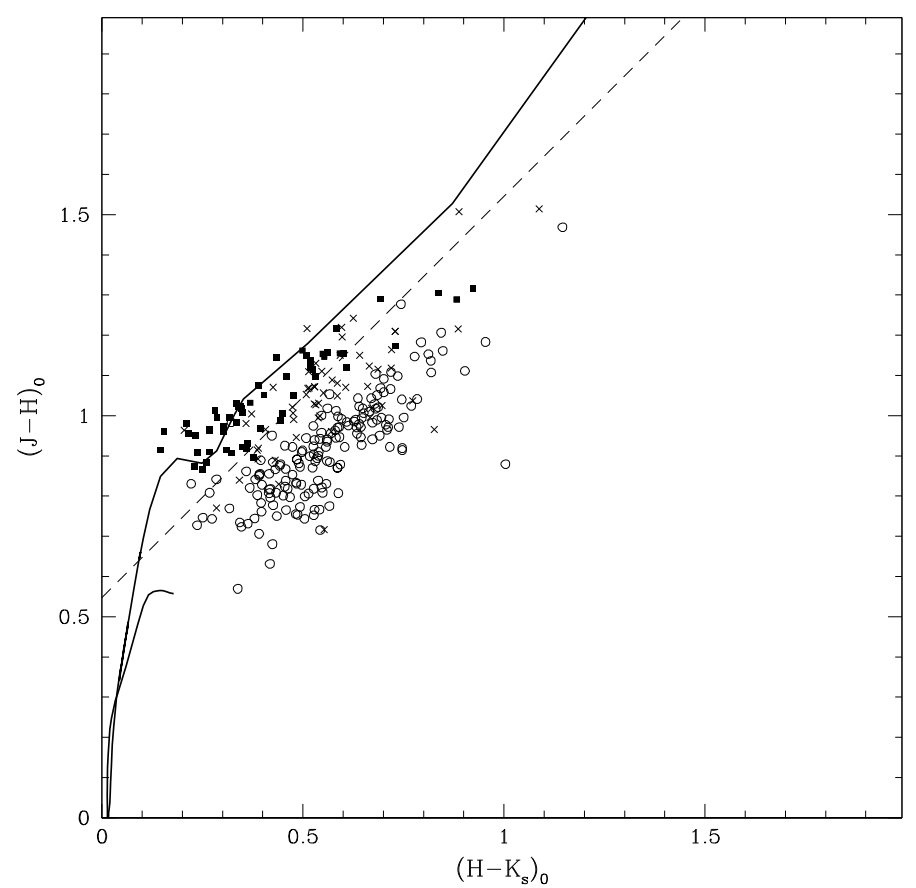

Fig. 7. Known C stars in WLM (black squares), IC 10 (crosses) and NGC 6822 (circles) are clearly separated. The dashed line represents Whitelock et al. (2006) regression. The curve is a typical $1 \mathrm{Gyr}$ isochrone.

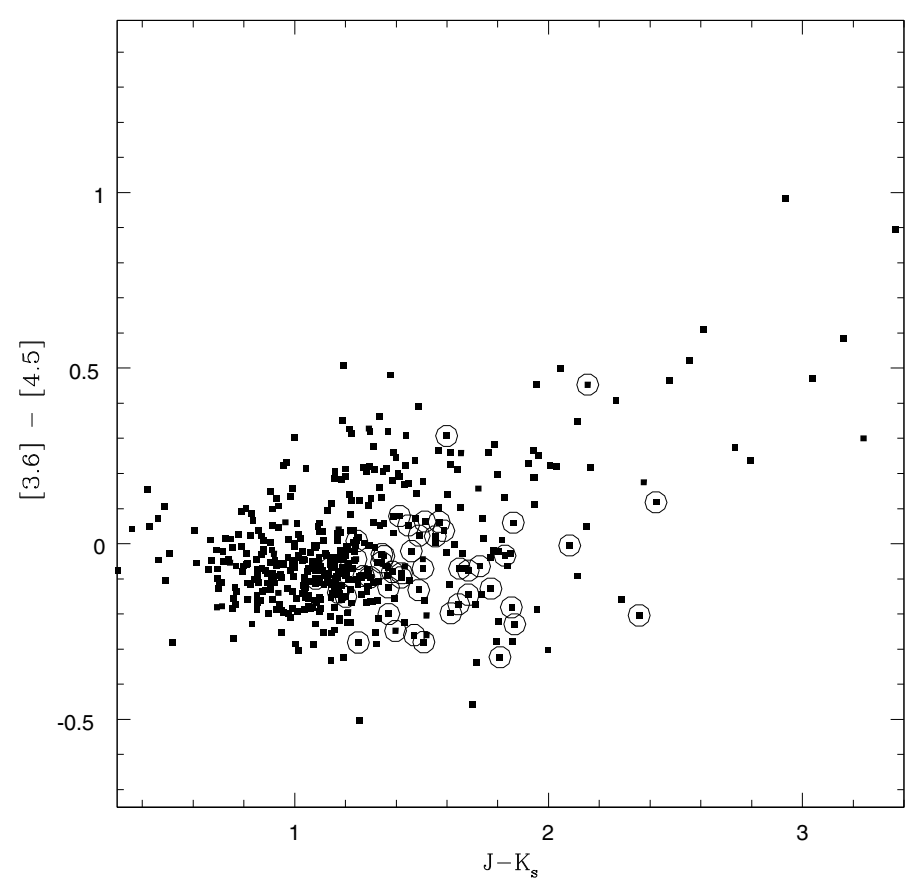

Fig. 8. Colour-colour diagram of the AGB stars in IC 1613. The circled dots are $\mathrm{C}$ stars identified by the narrow-band technique.

\section{Conclusion}

The photometric approach to identifying $\mathrm{C}$ stars from $\mathrm{M}$ giants leads to different results when one adopts the optical narrowband rather than the NIR colours.

The $(\mathrm{CN}-\mathrm{TiO})$ narrow-band technique has been successful in separating $\mathrm{C}$ stars and $\mathrm{M}$ giants, providing that their $(R-I)_{0}$ colour is higher than 0.90 . In that colour range one finds the $\mathrm{N}$-type C stars. The blue hotter C stars cannot be isolated from 
late $\mathrm{K}$ or early $\mathrm{M}$ stars because they have nearly the same $(\mathrm{CN}-$ $\mathrm{TiO})$ index.

The N-type C stars, however, show a range of $\left(J-K_{\mathrm{s}}\right)$ colours, overlapping substantially with $\mathrm{M}$ stars. Comparison of the $\mathrm{C}$ and $\mathrm{M}$ sample, obtained from the $(\mathrm{CN}-\mathrm{TiO})$, reveals, however, that very few $\mathrm{M}$ stars have $\left(J-K_{\mathrm{s}}\right)>1.4$, as can be seen in Fig. 6. Thus, this limit can be adopted as a working threshold for the detection of $\mathrm{C}$ stars. The determination of the $\mathrm{C} / \mathrm{M}$ ratio on the basis of the $\left(J-K_{\mathrm{s}}\right)$ colour selection of AGB stars leads to a ratio that differs from the one calculated from the narrowband identification. Furthermore, the determination of the number of AGB M stars requires the adoption of a blue colour limit in $\left(J-K_{\mathrm{s}}\right)$ to exclude the K-type AGB stars.

The effect of the metallicity on the above threshold is not yet fully demonstrated because our small galaxy sample does not cover a wide range of abundances. In this respect, the comparison of our narrow band survey of the low metallicity Sagittarius dwarf irregular galaxy (Demers \& Battinelli 2002) with the recently published NIR observations of Gullieuszik et al. (2007) could be useful to see if a metallicity effect is present. At the other abundance extreme, the narrow band survey of the outer disk of M 31 by Battinelli \& Demers (2003) should be repeated in the NIR.

Acknowledgements. This research is funded by the Natural Sciences and Engineering Research Council of Canada. We are grateful to Dr. Dale Jackson for providing the Spitzer data.

\section{References}

Albert, L., Demers, S., \& Kunkel, W. E. 2000, AJ, 119, 2780 Alonso, M. V., Minniti, D., Zijlstra, A. A., \& Tolstoy, E. 1999, A\&A, 346, 33
Battinelli, P., \& Demers, S. 2003, AJ, 125, 1298

Battinelli, P., \& Demers, S. 2005, A\&A, 434, 657

Battinelli, P., Demers, S., \& Mannucci, F. 2007, A\&A, 474, 35 (Paper I)

Bellazzini, M., Ferraro, F. R., Sollima, A., et al. 2004, A\&A, 424, 199

Brewer, J. P., Richer, H. B., \& Crabtree, D. R. 1996, AJ, 112, 491

Borissova, J., Georgiev, L., Kurtev, R., et al. 2000, Rev. Mex. Astron. Astrofis., 36,151

Cioni, M.-R. L., \& Habing, H. J. 2003, A\&A, 402, 133

Cioni, M.-R. L., \& Habing, H. J. 2005, A\&A, 429, 837

Cioni, M.-R. L., Girardi, L., Marigo, P., \& Habing, H. J. 2006, A\&A, 448, 77

Davidge, T. J. 2003, ApJ, 597, 289

Demers, S., \& Battinelli, P. 2002, AJ, 123, 238

Demers, S., Battinelli, P., \& Letarte, B. 2003, A\&A, 410, 795

Dolphin, A. E., Saha, A., Skillman, E. D., et al. 2001, ApJ, 550, 554

Dolphin, A., et al. 2005, see

http://purcell.as.arizona.edu/pubs/cancun.ps.gz

Gullieuszik, M., Rejkuba, M., Cioni, M. R., Habing, H. J., \& Held, E. V. 2007, A\&A, 475, 467

Jackson, D. C., Skillman, E. D., Gehrz, R. D., et al. 2007a, ApJ, 656, 818

Jackson, D. C., Skillman, E. D., Gehrz, R. D., et al. 2007b, ApJ, 667, 891

Kang, A., Sohn, Y.-J., Kim, H.-L., et al. A\&A, 454, 717

Lee, M. G. 1993, ApJ, 408, 409

Marigo, P., Girardi, L., Bressan, A., et al. 2008, A\&A, 482, 883

Mendez, B., Davis, M., Moustakas, J., et al. 2002, AJ, 124, 213

Pietrzyński, G., Gieren, W., Soszyński, I., et al. 2006a, ApJ, 642, 216

Pietrzyński, G., Gieren, W., Udalski, A., et al. 2006b, ApJ, 648, 366

Skillman, E. D., Tolstoy, E., Cole, A. A., et al. 2003, ApJ, 596, 253

Soszyński, I., Geiren, W., Pietrzyński, G., et al. 2006, ApJ, 648, 375

Valcheva, A. T., Ivanov, V. D., Ovcharov, E. P., \& Nedialkov, P. L. 2007, A\&A, 466, 501

Whitelock, P. A., Feast, M. W., Marang, F., \& Groenewegen, M. A. T. 2006, MNRAS, 369, 751

Zucker, D. B., \& Wyder, T. K. 2004, in Origin and Evolution of the Elements, a Carnegie Observatories Astrophysics Series, 4, ed. A. McWilliam, \& M. Rauch (Pasadena: Carnegie Observatories, http://www.ociw.edu/ociw/ symposia/series/symposium4/proceedings.html 
Table 2. NIR magnitudes and colours of C stars in IC 1613.

\begin{tabular}{|c|c|c|c|c|c|c|}
\hline id & RA & Dec & $K_{s}$ & $\sigma_{K}$ & $J-K_{s}$ & $\sigma_{J-K}$ \\
\hline 39 & $1: 4: 30.50$ & $2: 6: 47.7$ & 17.516 & 0.015 & 1.336 & 0.021 \\
\hline 49 & $1: 4: 33.90$ & $2: 8: 31.3$ & 15.941 & 0.009 & 1.342 & 0.017 \\
\hline 50 & $1: 4: 34.00$ & $2: 6: 0.6$ & 16.627 & 0.012 & 1.615 & 0.021 \\
\hline 51 & $1: 4: 34.50$ & $2: 10: 9.5$ & 16.999 & 0.012 & 1.333 & 0.018 \\
\hline 54 & $1: 4: 35.30$ & $2: 6: 6.1$ & 16.942 & 0.012 & 1.352 & 0.020 \\
\hline 55 & $1: 4: 35.40$ & $2: 6: 54.4$ & 16.904 & 0.011 & 1.397 & 0.016 \\
\hline 56 & $1: 4: 35.60$ & $2: 8: 31.3$ & 16.371 & 0.009 & 1.650 & 0.017 \\
\hline 57 & $1: 4: 36.60$ & $2: 6: 39.7$ & 16.134 & 0.009 & 1.861 & 0.014 \\
\hline 60 & 1:4:37.10 & $2: 7: 47.4$ & 17.244 & 0.013 & 1.505 & 0.019 \\
\hline 62 & $1: 4: 37.40$ & $2: 7: 39.9$ & 17.168 & 0.011 & 1.471 & 0.018 \\
\hline 63 & $1: 4: 37.40$ & $2: 9: 40.1$ & 17.006 & 0.012 & 1.336 & 0.021 \\
\hline 65 & $1: 4: 38.50$ & $2: 8: 22.3$ & 16.091 & 0.008 & 2.083 & 0.016 \\
\hline 66 & $1: 4: 38.50$ & $2: 9: 40.2$ & 16.620 & 0.011 & 1.406 & 0.020 \\
\hline 67 & $1: 4: 38.80$ & $2: 8: 54.0$ & 17.662 & 0.012 & 1.421 & 0.019 \\
\hline 68 & $1: 4: 39.00$ & $2: 8: 45.1$ & 17.073 & 0.014 & 1.490 & 0.025 \\
\hline 69 & $1: 4: 39.50$ & $2: 10: 10.4$ & 17.006 & 0.013 & 1.518 & 0.020 \\
\hline 70 & 1:4:39.80 & $2: 9: 13.9$ & 16.900 & 0.011 & 1.344 & 0.017 \\
\hline 71 & 1:4:39.90 & $2: 9: 52.7$ & 17.436 & 0.011 & 1.492 & 0.018 \\
\hline 73 & 1:4:40.10 & $2: 7: 40.0$ & 16.062 & 0.010 & 1.729 & 0.018 \\
\hline 74 & 1:4:40.10 & $2: 8: 45.4$ & 16.844 & 0.011 & 1.646 & 0.018 \\
\hline 76 & $1: 4: 40.70$ & $2: 7: 25.4$ & 17.191 & 0.011 & 1.419 & 0.018 \\
\hline 78 & $1: 4: 41.00$ & $2: 7: 7.1$ & 17.094 & 0.015 & 1.509 & 0.023 \\
\hline 79 & 1:4:41.70 & $2: 9: 57.6$ & 17.296 & 0.013 & 1.299 & 0.020 \\
\hline 81 & $1: 4: 41.90$ & $2: 8: 36.9$ & 17.665 & 0.013 & 1.289 & 0.019 \\
\hline 82 & $1: 4: 41.90$ & $2: 10: 2.5$ & 16.731 & 0.010 & 1.638 & 0.017 \\
\hline 85 & $1: 4: 42.30$ & $2: 8: 16.9$ & 16.737 & 0.010 & 1.773 & 0.018 \\
\hline 86 & $1: 4: 42.50$ & $2: 10: 26.0$ & 17.586 & 0.013 & 1.426 & 0.021 \\
\hline 88 & $1: 4: 42.70$ & $2: 8: 56.6$ & 15.515 & 0.009 & 2.357 & 0.016 \\
\hline 89 & $1: 4: 43.30$ & $2: 2: 9.7$ & 16.887 & 0.016 & 1.245 & 0.019 \\
\hline 90 & 1:4:43.30 & $2: 6: 4.5$ & 17.174 & 0.013 & 1.202 & 0.019 \\
\hline 92 & $1: 4: 43.60$ & $2: 8: 20.1$ & 16.295 & 0.010 & 1.867 & 0.017 \\
\hline 93 & $1: 4: 44.40$ & 2:8:9.7 & 16.585 & 0.009 & 1.809 & 0.016 \\
\hline 94 & $1: 4: 44.50$ & $2: 3: 44.5$ & 16.714 & 0.013 & 2.154 & 0.022 \\
\hline 95 & $1: 4: 44.70$ & $2: 7: 11.9$ & 17.497 & 0.012 & 1.554 & 0.019 \\
\hline 97 & $1: 4: 45.40$ & $2: 4: 38.3$ & 17.169 & 0.018 & 1.172 & 0.022 \\
\hline 98 & 1:4:46.00 & $2: 10: 28.3$ & 17.719 & 0.013 & 1.306 & 0.020 \\
\hline 99 & 1:4:47.30 & $2: 8: 36.0$ & 16.252 & 0.007 & 1.802 & 0.011 \\
\hline 100 & $1: 4: 47.70$ & $2: 6: 29.0$ & 17.041 & 0.011 & 1.242 & 0.017 \\
\hline 101 & $1: 4: 47.70$ & $2: 8: 15.4$ & 15.897 & 0.008 & 1.854 & 0.016 \\
\hline 102 & 1:4:48.20 & $2: 6: 5.5$ & 16.709 & 0.012 & 1.599 & 0.021 \\
\hline 103 & $1: 4: 48.50$ & $2: 5: 16.3$ & 16.214 & 0.011 & 1.828 & 0.017 \\
\hline 104 & $1: 4: 49.20$ & $2: 3: 26.3$ & 16.851 & 0.013 & 1.370 & 0.018 \\
\hline 107 & $1: 4: 50.20$ & $2: 6: 39.0$ & 16.690 & 0.015 & 1.513 & 0.026 \\
\hline 108 & $1: 4: 50.30$ & $2: 4: 47.1$ & 15.923 & 0.011 & 1.448 & 0.015 \\
\hline 111 & $1: 4: 50.60$ & $2: 3: 42.1$ & 16.616 & 0.013 & 1.297 & 0.018 \\
\hline 113 & $1: 4: 51.40$ & $2: 10: 41.7$ & 16.775 & 0.010 & 1.236 & 0.013 \\
\hline 114 & 1:4:51.60 & $2: 10: 23.5$ & 17.691 & 0.012 & 1.319 & 0.019 \\
\hline 116 & 1:4:52.00 & $2: 4: 19.5$ & 17.312 & 0.019 & 1.569 & 0.027 \\
\hline 117 & $1: 4: 52.30$ & $2: 4: 13.4$ & 15.799 & 0.009 & 1.488 & 0.014 \\
\hline 118 & $1: 4: 52.50$ & $2: 4: 24.9$ & 16.445 & 0.013 & 1.684 & 0.019 \\
\hline 122 & $1: 4: 54.20$ & $2: 3: 44.9$ & 17.100 & 0.019 & 1.144 & 0.023 \\
\hline 123 & $1: 4: 54.30$ & $2: 4: 20.6$ & 17.043 & 0.017 & 1.460 & 0.023 \\
\hline 125 & 1:4:56.10 & $2: 7: 45.0$ & 16.845 & 0.009 & 1.587 & 0.014 \\
\hline 126 & $1: 4: 56.20$ & $2: 8: 2.9$ & 16.559 & 0.008 & 1.684 & 0.014 \\
\hline 128 & 1:4:57.90 & $2: 10: 44.4$ & 17.227 & 0.013 & 1.251 & 0.020 \\
\hline 129 & 1:4:58.10 & $2: 2: 57.7$ & 16.690 & 0.015 & 1.413 & 0.019 \\
\hline 131 & 1:4:58.60 & $2: 4: 26.5$ & 16.396 & 0.013 & 1.272 & 0.017 \\
\hline 134 & $1: 4: 59.30$ & $2: 6: 58.7$ & 17.559 & 0.012 & 1.083 & 0.017 \\
\hline 135 & 1:4:59.60 & $2: 9: 58.2$ & 16.862 & 0.010 & 1.371 & 0.015 \\
\hline 139 & 1:5:0.70 & $2: 7: 10.1$ & 17.282 & 0.011 & 2.423 & 0.027 \\
\hline 142 & $1: 5: 2.60$ & $2: 7: 17.8$ & 16.998 & 0.011 & 1.384 & 0.015 \\
\hline
\end{tabular}

Table 3. NIR magnitudes and colours of C stars in NGC 3109.

\begin{tabular}{|c|c|c|c|c|c|c|}
\hline id & RA & Dec & $K_{s}$ & $\sigma_{K}$ & $J-K_{s}$ & $\sigma_{J-K}$ \\
\hline 106 & $10: 2: 53.17$ & $-26: 9: 8.2$ & 18.023 & 0.031 & 1.255 & 0.048 \\
\hline 108 & $10: 2: 53.92$ & $-26: 10: 20.1$ & 17.550 & 0.037 & 1.531 & 0.056 \\
\hline 109 & $10: 2: 54.11$ & $-26: 10: 33.2$ & 17.514 & 0.043 & 1.532 & 0.056 \\
\hline 111 & $10: 2: 54.29$ & $-26: 9: 57.8$ & 18.843 & 0.040 & 1.163 & 0.047 \\
\hline 112 & $10: 2: 54.59$ & $-26: 10: 2.5$ & 18.081 & 0.025 & 1.513 & 0.029 \\
\hline 113 & $10: 2: 54.74$ & $-26: 8: 52.1$ & 18.477 & 0.035 & 1.260 & 0.043 \\
\hline 116 & $10: 2: 54.90$ & $-26: 10: 34.8$ & 18.586 & 0.038 & 1.278 & 0.048 \\
\hline 117 & $10: 2: 55.24$ & $-26: 9: 13.0$ & 18.302 & 0.023 & 1.154 & 0.026 \\
\hline 118 & $10: 2: 55.40$ & $-26: 9: 19.2$ & 18.868 & 0.033 & 1.153 & 0.038 \\
\hline 119 & $10: 2: 55.42$ & $-26: 10: 59.8$ & 17.997 & 0.038 & 1.382 & 0.054 \\
\hline 122 & $10: 2: 55.95$ & $-26: 8: 37.6$ & 18.403 & 0.045 & 1.108 & 0.060 \\
\hline 123 & $10: 2: 56.59$ & $-26: 8: 54.7$ & 17.477 & 0.021 & 1.797 & 0.028 \\
\hline 124 & $10: 2: 56.73$ & $-26: 9: 13.3$ & 17.747 & 0.020 & 1.501 & 0.033 \\
\hline 126 & $10: 2: 56.94$ & $-26: 9: 7.5$ & 17.497 & 0.025 & 2.814 & 0.033 \\
\hline 127 & $10: 2: 57.09$ & $-26: 9: 39.0$ & 17.659 & 0.018 & 1.869 & 0.024 \\
\hline 128 & $10: 2: 57.12$ & $-26: 9: 22.0$ & 18.738 & 0.033 & 2.168 & 0.048 \\
\hline 130 & $10: 2: 57.24$ & $-26: 9: 46.2$ & 18.353 & 0.022 & 1.428 & 0.032 \\
\hline 132 & $10: 2: 57.79$ & $-26: 8: 38.2$ & 17.535 & 0.032 & 1.990 & 0.043 \\
\hline 134 & $10: 2: 58.09$ & $-26: 9: 18.9$ & 17.016 & 0.020 & 1.715 & 0.033 \\
\hline 135 & $10: 2: 58.38$ & $-26: 8: 56.9$ & 17.318 & 0.016 & 1.809 & 0.023 \\
\hline 136 & $10: 2: 58.42$ & $-26: 10: 46.8$ & 18.347 & 0.029 & 1.297 & 0.034 \\
\hline 137 & $10: 2: 58.92$ & $-26: 9: 49.6$ & 18.002 & 0.024 & 1.446 & 0.036 \\
\hline 138 & $10: 2: 58.95$ & $-26: 9: 7.5$ & 19.133 & 0.038 & 1.033 & 0.045 \\
\hline 139 & $10: 2: 58.99$ & $-26: 9: 17.7$ & 18.673 & 0.018 & 1.148 & 0.023 \\
\hline 142 & $10: 2: 59.39$ & $-26: 10: 46.5$ & 17.744 & 0.029 & 1.712 & 0.036 \\
\hline 143 & $10: 2: 59.46$ & $-26: 8: 39.3$ & 17.321 & 0.022 & 1.412 & 0.030 \\
\hline 145 & $10: 2: 59.49$ & $-26: 9: 18.8$ & 17.315 & 0.018 & 1.805 & 0.027 \\
\hline 146 & 10:3:0.01 & $-26: 8: 54.1$ & 18.124 & 0.027 & 1.280 & 0.034 \\
\hline 147 & 10:3:0.02 & $-26: 8: 44.3$ & 16.686 & 0.022 & 1.858 & 0.030 \\
\hline 148 & $10: 3: 0.07$ & $-26: 10: 12.2$ & 18.603 & 0.031 & 1.239 & 0.036 \\
\hline 150 & $10: 3: 0.47$ & $-26: 8: 37.2$ & 17.478 & 0.037 & 1.629 & 0.062 \\
\hline 151 & $10: 3: 0.70$ & $-26: 10: 12.7$ & 17.126 & 0.019 & 1.668 & 0.030 \\
\hline 152 & $10: 3: 0.77$ & $-26: 8: 56.0$ & 17.136 & 0.018 & 1.208 & 0.028 \\
\hline 154 & $10: 3: 0.89$ & $-26: 8: 47.6$ & 17.266 & 0.019 & 1.440 & 0.026 \\
\hline 156 & $10: 3: 1.01$ & $-26: 8: 43.2$ & 18.571 & 0.027 & 1.287 & 0.038 \\
\hline 157 & $10: 3: 1.11$ & $-26: 9: 19.2$ & 18.216 & 0.025 & 1.625 & 0.035 \\
\hline 158 & $10: 3: 1.13$ & $-26: 8: 47.3$ & 17.847 & 0.018 & 1.415 & 0.031 \\
\hline 159 & $10: 3: 1.43$ & $-26: 8: 51.7$ & 17.525 & 0.018 & 1.281 & 0.024 \\
\hline 160 & $10: 3: 1.49$ & $-26: 10: 23.4$ & 18.543 & 0.032 & 1.233 & 0.041 \\
\hline 161 & $10: 3: 1.52$ & $-26: 9: 1.2$ & 17.101 & 0.023 & 1.640 & 0.029 \\
\hline 163 & $10: 3: 1.80$ & $-26: 9: 32.5$ & 18.282 & 0.031 & 1.212 & 0.038 \\
\hline 164 & $10: 3: 1.86$ & $-26: 8: 51.0$ & 17.830 & 0.031 & 1.973 & 0.049 \\
\hline 165 & 10:3:1.98 & $-26: 10: 32.7$ & 18.330 & 0.041 & 1.538 & 0.049 \\
\hline 166 & $10: 3: 2.01$ & $-26: 9: 10.6$ & 17.798 & 0.021 & 1.814 & 0.029 \\
\hline 168 & $10: 3: 2.46$ & $-26: 9: 33.4$ & 17.928 & 0.019 & 1.519 & 0.027 \\
\hline 169 & $10: 3: 2.59$ & $-26: 8: 41.9$ & 17.907 & 0.040 & 1.604 & 0.051 \\
\hline 170 & $10: 3: 2.65$ & $-26: 9: 22.3$ & 17.289 & 0.020 & 1.590 & 0.027 \\
\hline 170 & $10: 3 \cdot 2.65$ & $-26: 9 \cdot 22.3$ & 17.516 & 0.033 & 1.454 & 0.045 \\
\hline 171 & $10: 3: 2.69$ & $-26: 8: 45.8$ & 18.745 & 0.046 & 1.152 & 0.059 \\
\hline 172 & $10: 3: 2.74$ & $-26: 9: 11.4$ & 17.379 & 0.032 & 1.413 & 0.044 \\
\hline 176 & $10: 3: 3.14$ & $-26: 8: 55.4$ & 18.135 & 0.037 & 1.259 & 0.050 \\
\hline 177 & $10: 3: 3.19$ & $-26: 10: 18.6$ & 19.067 & 0.032 & 1.256 & 0.045 \\
\hline 177 & $10: 3: 3.19$ & $-26: 10: 18.6$ & 19.170 & 0.054 & 1.234 & 0.061 \\
\hline 178 & $10: 3: 3.21$ & $-26: 9: 43.4$ & 18.826 & 0.030 & 1.277 & 0.037 \\
\hline 179 & $10: 3: 3.28$ & $-26: 9: 34.1$ & 18.826 & 0.046 & 1.276 & 0.057 \\
\hline 180 & $10: 3: 3.31$ & $-26: 9: 1.8$ & 17.747 & 0.033 & 1.576 & 0.043 \\
\hline 180 & $10: 3: 3.31$ & $-26: 9: 1.8$ & 17.809 & 0.045 & 1.591 & 0.063 \\
\hline 181 & $10: 3: 3.38$ & $-26: 9: 43.5$ & 18.048 & 0.021 & 1.275 & 0.026 \\
\hline 181 & $10: 3: 3.38$ & $-26: 9: 43.5$ & 18.133 & 0.036 & 1.212 & 0.045 \\
\hline 182 & $10: 3: 3.53$ & $-26: 9: 58.6$ & 16.647 & 0.022 & 1.599 & 0.033 \\
\hline 182 & $10: 3: 3.53$ & $-26: 9: 58.6$ & 16.770 & 0.039 & 1.448 & 0.047 \\
\hline 183 & $10: 3: 3.53$ & $-26: 10: 12.3$ & 17.690 & 0.026 & 1.671 & 0.033 \\
\hline 183 & $10: 3: 3.53$ & $-26: 10: 12.3$ & 17.795 & 0.025 & 1.672 & 0.033 \\
\hline 184 & $10: 3: 3.66$ & $-26: 9: 15.7$ & 17.871 & 0.036 & 1.366 & 0.041 \\
\hline 185 & $10: 3: 3.95$ & $-26: 8: 58.2$ & 17.390 & 0.052 & 1.603 & 0.066 \\
\hline
\end{tabular}


Table 3. continued.

Table 3. continued.

\begin{tabular}{|c|c|c|c|c|c|c|}
\hline id & RA & Dec & $\overline{K_{s}}$ & $\sigma_{K}$ & $J-K_{s}$ & $\sigma_{J-K}$ \\
\hline 185 & $10: 3: 3.95$ & $-26: 8: 58.2$ & 17.473 & 0.065 & 1.545 & 0.076 \\
\hline 187 & $10: 3: 4.14$ & $-26: 8: 34.8$ & 18.120 & 0.059 & 1.231 & 0.090 \\
\hline 188 & $10: 3: 4.27$ & $-26: 8: 56.6$ & 18.417 & 0.032 & 1.207 & 0.051 \\
\hline 191 & $10: 3: 4.59$ & $-26: 9: 38.3$ & 17.859 & 0.035 & 1.216 & 0.044 \\
\hline 192 & $10: 3: 5.01$ & $-26: 9: 0.0$ & 17.460 & 0.047 & 1.752 & 0.055 \\
\hline 196 & $10: 3: 5.74$ & $-26: 11: 24.9$ & 17.325 & 0.035 & 1.247 & 0.046 \\
\hline 197 & 10:3:6.19 & $-26: 8: 57.4$ & 17.501 & 0.050 & 1.771 & 0.059 \\
\hline 199 & $10: 3: 6.42$ & $-26: 9: 0.3$ & 17.362 & 0.043 & 1.933 & 0.049 \\
\hline 203 & 10:3:8.07 & $-26: 9: 4.8$ & 17.410 & 0.034 & 1.975 & 0.037 \\
\hline 204 & $10: 3: 8.13$ & $-26: 10: 40.7$ & 18.088 & 0.038 & 1.912 & 0.046 \\
\hline 205 & $10: 3: 8.28$ & $-26: 9: 18.6$ & 17.402 & 0.027 & 1.206 & 0.032 \\
\hline 208 & $10: 3: 8.42$ & $-26: 9: 6.1$ & 17.368 & 0.035 & 1.829 & 0.043 \\
\hline 209 & 10:3:8.98 & $-26: 10: 24.2$ & 17.863 & 0.029 & 1.427 & 0.033 \\
\hline 211 & 10:3:8.99 & $-26: 10: 2.3$ & 17.826 & 0.039 & 2.425 & 0.043 \\
\hline 213 & 10:3:9.10 & $-26: 8: 59.9$ & 17.784 & 0.039 & 1.603 & 0.047 \\
\hline 216 & 10:3:9.41 & $-26: 9: 8.5$ & 17.756 & 0.030 & 1.458 & 0.038 \\
\hline 217 & $10: 3: 9.51$ & $-26: 10: 26.6$ & 17.429 & 0.026 & 1.760 & 0.030 \\
\hline 218 & $10: 3: 9.53$ & $-26: 10: 33.3$ & 17.463 & 0.029 & 1.587 & 0.032 \\
\hline 219 & 10:3:9.56 & $-26: 9: 1.2$ & 18.109 & 0.032 & 1.418 & 0.037 \\
\hline 222 & 10:3:9.70 & $-26: 9: 13.1$ & 16.850 & 0.029 & 1.331 & 0.034 \\
\hline 223 & 10:3:9.88 & $-26: 9: 16.6$ & 18.250 & 0.024 & 1.213 & 0.033 \\
\hline 224 & 10:3:9.91 & $-26: 10: 17.5$ & 18.015 & 0.039 & 1.274 & 0.047 \\
\hline 225 & 10:3:10.11 & $-26: 9: 22.8$ & 16.875 & 0.024 & 1.637 & 0.030 \\
\hline 226 & $10: 3: 10.15$ & $-26: 9: 31.1$ & 18.577 & 0.027 & 1.121 & 0.035 \\
\hline 228 & $10: 3: 10.44$ & $-26: 10: 31.8$ & 17.552 & 0.034 & 1.500 & 0.038 \\
\hline 229 & $10: 3: 10.68$ & $-26: 10: 17.1$ & 17.736 & 0.022 & 1.698 & 0.029 \\
\hline 230 & $10: 3: 10.75$ & $-26: 9: 31.2$ & 18.244 & 0.025 & 1.469 & 0.033 \\
\hline 232 & $10: 3: 10.96$ & $-26: 9: 17.0$ & 17.950 & 0.034 & 1.207 & 0.040 \\
\hline 233 & $10: 3: 11.07$ & $-26: 9: 16.1$ & 17.813 & 0.030 & 1.724 & 0.035 \\
\hline 236 & $10: 3: 11.40$ & $-26: 9: 11.8$ & 17.273 & 0.047 & 1.489 & 0.051 \\
\hline 237 & $10: 3: 11.41$ & $-26: 9: 25.6$ & 18.158 & 0.048 & 1.691 & 0.053 \\
\hline 239 & 10:3:11.49 & $-26: 9: 23.8$ & 17.840 & 0.031 & 1.713 & 0.037 \\
\hline 240 & $10: 3: 11.78$ & $-26: 10: 39.7$ & 17.943 & 0.038 & 2.019 & 0.048 \\
\hline 241 & $10: 3: 11.86$ & $-26: 8: 57.8$ & 17.614 & 0.077 & 1.629 & 0.087 \\
\hline 242 & $10: 3: 11.87$ & $-26: 10: 14.8$ & 17.240 & 0.026 & 1.646 & 0.032 \\
\hline 243 & $10: 3: 11.97$ & $-26: 9: 38.3$ & 17.221 & 0.026 & 1.357 & 0.032 \\
\hline 244 & $10: 3: 11.98$ & $-26: 9: 18.4$ & 17.565 & 0.037 & 1.950 & 0.043 \\
\hline 245 & 10:3:11.99 & $-26: 9: 50.3$ & 17.656 & 0.032 & 1.621 & 0.040 \\
\hline 247 & $10: 3: 12.39$ & $-26: 9: 35.7$ & 16.910 & 0.030 & 1.811 & 0.036 \\
\hline 249 & $10: 3: 12.67$ & $-26: 9: 45.6$ & 18.671 & 0.041 & 1.206 & 0.049 \\
\hline 251 & $10: 3: 12.82$ & $-26: 9: 31.2$ & 17.028 & 0.046 & 1.642 & 0.053 \\
\hline 252 & $10: 3: 12.84$ & $-26: 9: 33.8$ & 17.961 & 0.054 & 1.515 & 0.060 \\
\hline 253 & $10: 3: 12.84$ & $-26: 9: 49.3$ & 17.772 & 0.043 & 1.529 & 0.048 \\
\hline 254 & $10: 3: 12.90$ & $-26: 9: 1.5$ & 17.400 & 0.089 & 2.031 & 0.107 \\
\hline 256 & 10:3:12.97 & $-26: 9: 15.6$ & 17.268 & 0.069 & 1.781 & 0.077 \\
\hline 257 & $10: 3: 13.10$ & $-26: 10: 48.4$ & 17.965 & 0.046 & 2.719 & 0.058 \\
\hline 258 & $10: 3: 13.12$ & $-26: 9: 28.0$ & 16.384 & 0.069 & 1.893 & 0.078 \\
\hline 259 & $10: 3: 13.18$ & $-26: 9: 26.7$ & 17.382 & 0.049 & 1.740 & 0.061 \\
\hline 260 & $10: 3: 13.35$ & $-26: 9: 52.6$ & 17.344 & 0.022 & 1.640 & 0.039 \\
\hline 261 & $10: 3: 13.36$ & $-26: 10: 30.6$ & 17.483 & 0.039 & 1.914 & 0.048 \\
\hline 261 & $10: 3: 13.36$ & $-26: 10: 30.6$ & 17.495 & 0.036 & 1.903 & 0.050 \\
\hline 262 & $10: 3: 13.43$ & $-26: 9: 14.0$ & 18.121 & 0.084 & 1.698 & 0.099 \\
\hline 265 & $10: 3: 13.84$ & $-26: 10: 12.7$ & 16.742 & 0.029 & 1.767 & 0.040 \\
\hline 268 & $10: 3: 14.10$ & $-26: 10: 38.8$ & 17.783 & 0.045 & 1.776 & 0.056 \\
\hline 269 & $10: 3: 14.12$ & $-26: 10: 15.4$ & 17.044 & 0.029 & 1.845 & 0.040 \\
\hline 273 & $10: 3: 14.92$ & $-26: 10: 20.6$ & 17.183 & 0.020 & 1.489 & 0.031 \\
\hline 274 & $10: 3: 14.95$ & $-26: 9: 46.6$ & 18.113 & 0.080 & 1.393 & 0.103 \\
\hline 275 & $10: 3: 15.10$ & $-26: 10: 4.7$ & 18.074 & 0.026 & 1.343 & 0.044 \\
\hline 276 & $10: 3: 15.35$ & $-26: 10: 39.9$ & 18.368 & 0.027 & 1.788 & 0.040 \\
\hline 281 & $10: 3: 15.72$ & $-26: 10: 38.4$ & 17.781 & 0.048 & 1.562 & 0.062 \\
\hline 283 & $10: 3: 15.89$ & $-26: 10: 3.9$ & 17.156 & 0.026 & 1.836 & 0.037 \\
\hline
\end{tabular}

\begin{tabular}{lcccccc}
\hline id & RA & Dec & $K_{s}$ & $\sigma_{K}$ & $J-K_{s}$ & $\sigma_{J-K}$ \\
\hline 285 & $10: 3: 15.98$ & $-26: 9: 21.6$ & 17.394 & 0.017 & 1.770 & 0.085 \\
287 & $10: 3: 16.08$ & $-26: 10: 24.7$ & 17.325 & 0.029 & 2.110 & 0.037 \\
288 & $10: 3: 16.12$ & $-26: 10: 49.3$ & 17.695 & 0.026 & 1.829 & 0.039 \\
291 & $10: 3: 16.48$ & $-26: 9: 35.3$ & 18.807 & 0.072 & 0.933 & 0.090 \\
295 & $10: 3: 17.06$ & $-26: 9: 44.2$ & 17.903 & 0.024 & 1.234 & 0.036 \\
297 & $10: 3: 17.28$ & $-26: 10: 27.4$ & 18.153 & 0.025 & 1.597 & 0.040 \\
304 & $10: 3: 18.07$ & $-26: 10: 8.1$ & 17.637 & 0.026 & 2.208 & 0.049 \\
308 & $10: 3: 18.43$ & $-26: 11: 1.8$ & 17.233 & 0.023 & 1.821 & 0.031 \\
311 & $10: 3: 18.61$ & $-26: 10: 27.1$ & 17.726 & 0.020 & 2.135 & 0.035 \\
316 & $10: 3: 19.05$ & $-26: 9: 23.1$ & 17.057 & 0.020 & 2.195 & 0.060 \\
324 & $10: 3: 19.95$ & $-26: 8: 58.4$ & 17.394 & 0.018 & 2.167 & 0.028 \\
325 & $10: 3: 20.03$ & $-26: 10: 9.0$ & 17.120 & 0.019 & 1.848 & 0.039 \\
327 & $10: 3: 20.07$ & $-26: 10: 10.1$ & 17.001 & 0.016 & 1.529 & 0.032 \\
328 & $10: 3: 20.12$ & $-26: 10: 11.1$ & 18.478 & 0.023 & 1.553 & 0.056 \\
329 & $10: 3: 20.16$ & $-26: 10: 31.1$ & 17.389 & 0.023 & 2.146 & 0.049 \\
331 & $10: 3: 20.66$ & $-26: 10: 2.7$ & 17.446 & 0.018 & 1.819 & 0.027 \\
340 & $10: 3: 21.76$ & $-26: 10: 2.7$ & 17.602 & 0.024 & 1.586 & 0.039 \\
341 & $10: 3: 21.86$ & $-26: 10: 41.9$ & 17.931 & 0.025 & 2.075 & 0.037 \\
342 & $10: 3: 21.89$ & $-26: 11: 4.9$ & 17.253 & 0.048 & 2.093 & 0.057 \\
343 & $10: 3: 22.05$ & $-26: 9: 33.5$ & 18.431 & 0.033 & 1.276 & 0.042 \\
344 & $10: 3: 22.18$ & $-26: 10: 17.0$ & 17.825 & 0.019 & 1.701 & 0.030 \\
345 & $10: 3: 22.23$ & $-26: 9: 11.9$ & 17.067 & 0.026 & 2.059 & 0.036 \\
348 & $10: 3: 22.40$ & $-26: 10: 4.0$ & 17.845 & 0.025 & 1.626 & 0.033 \\
354 & $10: 3: 22.91$ & $-26: 10: 10.7$ & 18.130 & 0.016 & 1.753 & 0.032 \\
357 & $10: 3: 23.37$ & $-26: 10: 2.8$ & 18.453 & 0.027 & 1.233 & 0.041 \\
358 & $10: 3: 23.74$ & $-26: 11: 9.0$ & 18.421 & 0.062 & 1.633 & 0.100 \\
360 & $10: 3: 23.85$ & $-26: 10: 17.7$ & 18.657 & 0.026 & 1.472 & 0.043 \\
361 & $10: 3: 23.95$ & $-26: 10: 34.1$ & 18.107 & 0.042 & 1.781 & 0.058 \\
364 & $10: 3: 24.08$ & $-26: 10: 40.2$ & 17.842 & 0.036 & 1.610 & 0.048 \\
366 & $10: 3: 24.37$ & $-26: 8: 54.1$ & 18.712 & 0.070 & 1.179 & 0.083 \\
367 & $10: 3: 24.49$ & $-26: 10: 52.1$ & 18.145 & 0.049 & 1.658 & 0.085 \\
\hline & & & & & &
\end{tabular}

